\title{
Burn Wound Successfully Treated with 830-nm Light Emitting Diode Phototherapy Combined with Epidermal Growth Factor Solution
}

\author{
Hae-Jin Lee ${ }^{1}$ \\ Young Koo Kim² \\ ${ }^{1}$ Yonsei A\&B Clinic of Dermatology, Seoul, Korea \\ ${ }^{2}$ Yonsei Star Skin \& Laser Clinic, Seoul, Korea
}

\begin{abstract}
Burns are one of the most extensive injuries of soft tissues as well as skin, occasionally resulting in extensive, deep wounds and death. Burn wounds can lead to severe physical and psychological distress because of excessive scarring and skin contractures. Treatment of burn wounds has always been a challenging problem and many different methods have been used to treat such injuries. We report here on treating a patient with a burn wound using 830-nm light emitting diode (LED) phototherapy combined with epidermal growth factor (EGF) solution. After five daily sessions of LED with EGF solution treatment, the patient demonstrated nearly complete improvement with no remarkable side effects. We suggest that LED phototherapy combined with EGF solution could be an effective and safe treatment option for treating burn wounds.
\end{abstract}

\section{Key words}

Burn wound; Burn scar; Epidermal growth factor; Light emitting diode phototherapy; Low-level light therapy 


\section{INTRODUCTION}

Acute thermal injuries requiring medical treatment affect 67 percent of people in lower middle socioeconomic countries of Africa and South-East Asia regions.' Burn wounds can also lead to post-injury scar tissue formation with long-term functional and psychosocial consequences. ${ }^{2}$ Especially in diabetic patients, impaired wound healing is concomitant with complex pathological phenomenon, and the prolonged inflammation along with increased oxidative stress impairs healing process. ${ }^{1}$ Treatment of diabetic burn wounds has always been a challenging problem and many different methods have been used to treat such injuries, which should include blood sugar control, removal of wound exudate and dead tissue, early resuscitation, wound excision and coverage, infection management and proper choice of wound dressings. ${ }^{2,3}$ Several studies have reported the development of increasingly efficient approaches for tissue repair, with minimization of the factors that delay or impede the healing process in burn wounds which including applicating epidermal growth factor (EGF)-containing formulations in and/or around lesions, and light-emitting diode (LED) therapy. ${ }^{1,4}$ Because of situations mentioned above, many clinicians have a tendency to hesitate treating with single modality in burn wound with various medical conditions. When deciding combination treatment in burn wound, clinicians should consider the synergistic effects between each modalities.

Herein, we report a case of patient with burn wound using 830-nm light emitting diode (LED) phototherapy combined with epidermal growth factor (EGF) solution. After five sessions of wound management, the patient presented nearly complete improvement in the burn lesions with no remarkable side effects or recurrence over the duration of 3 months.

\section{CASE REPORT}

A 71-year-old, Korean woman visited our clinic with irregular shaped ulcer with erosion wound on the left lower leg due to hot water (Fig. 1A). The patient had 10 years of history of type 2 diabetes mellitus (DM), which is properly controlled by DM medication. The patient was clinically diagnosed with second degree burn (Fig. 1A) and was scheduled to undergo LED treatment combined with applying EGF solution. After obtaining written informed consent, the patient was treated with every other day scheduled, 5 sessions of 830-nm LED phototherapy (HEALITE; Lutronic Corp., Goyang, Korea) was performed at the treatment settings of 20 minutes of exposure with $15 \mathrm{~cm}$ distance on the skin lesion. The treated areas were occluded and applied with $0.5 \mathrm{ml}$ of 4 ppm EGF solutions (PEPS mist; TNH BIO, Suwon, Koreal without systemic or topical antibiotics.

At 10 days after the first treatment of LED with EGF solution, the patient presented nearly complete improvement with minimal erythema and postinflammatory hyperpigmentation without worsening or recurrence (Fig. 1B).

\section{DISCUSSION}

Burn wounds are one of the most extensive injuries of soft tissues as well as skin occasionally resulting in extensive and deep wounds and death. ${ }^{2}$ Tissue damage in burn wound triggers a cascade of biochemical and molecular events that initiate a rapid but highly complex tissue repair process. ${ }^{5}$ Recent study revealed the positive therapeutic effect of treating burn wounds with LED device and EGF solution ${ }^{1,4}$, but there is lack of study with synergistic effect of modalities mentioned above.

LED can reduce the inflammatory response by blocking the effects of ROS via activation of IL-6 and inhibition of cell proliferation and differentiation induced by growth factors via the MAPK pathway in burn wound model. ${ }^{4} \mathrm{Ap}$ plication of EGF-containing formulations around lesions is widely used in wound treatments. Sen et al. ${ }^{1}$ reported applying EGF solution showed faster granulation, enhanced collagenization, patterned re-epithelialization and

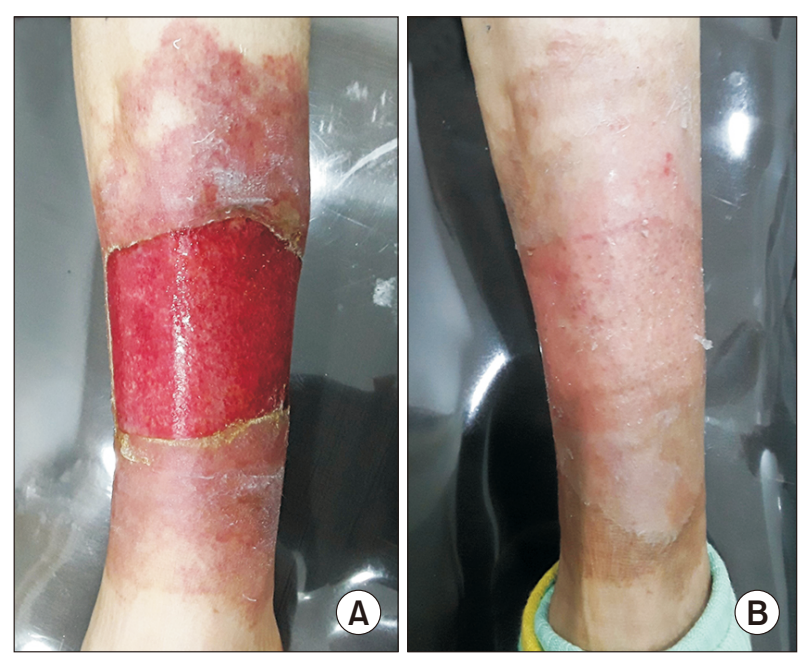

Fig. 1. Photographs of a 71-year-old, Koream female with second degree burn (A) at baseline, (B) after every other day scheduled, 5 sessions of 830-nm light emitting diode phototherapy combined with epidermal growth factor solution (10 days after baseline). 


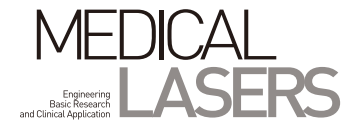

immunomodulatory effect in diabetic burn wound model. Therefore, many clinicians widely used LED and EGF solutions in treating various wounds including burn wound.

Although most of 2nd degree burn wounds are treated with two weeks, if properly treated, delayed wound healing might cause several side effects including scarring and contracture formation with significant functional and esthetic problems. Many patients would have pruritus and neuropathic pain if burns are deep. ${ }^{6}$ In our case, total ten days of treatment is needed to finish the reepithelization of wound with minimal erythema and postinflammatory hyperpigmentation although DM might delay the wound healing process. The authors might suggest that LED phototherapy and applying EGF solution could have synergistic effect in wound healing via immunomudulatory mechanism. Further controlled study is needed to clarify the synergistic mechanism between LED phototherapy and EGF on wound healing.

In conclusion, the authors suggest that $830-n m$ LED phototherapy combined with EGF solution could be effective and safe treatment option in treating the patient with second degree burn wound. Further prospective, controlled investigations are needed to be followed to compare the synergistic effect of LED combined with EGF solution to optimize the treatment settings for burn wound.

\section{CONFLICT OF INTEREST}

The authors have no conflict of interest to disclose.

\section{REFERENCES}

1. Sen S, Basak P, Prasad Sinha B, Maurye P, Kumar Jaiswal K, et al. Anti-inflammatory effect of epidermal growth factor conjugated silk fibroin immobilized polyurethane ameliorates diabetic burn wound healing. Int J Biol Macromol In press 2019.

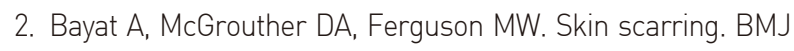
2003;326:88-92.

3. Jeschke MG, Rehou S, McCann MR, Shahrokhi S. Allogeneic mesenchymal stem cells for treatment of severe burn injury. Stem Cell Res Ther 2019;10:337.

4. Silveira PC, Ferreira KB, da Rocha FR, Pieri BL, Pedroso GS, De Souza CT, et al. Effect of low-power laser (LPL) and lightemitting diode (LED) on inflammatory response in burn wound healing. Inflammation 2016;39:1395-404.

5. Martin P, Leibovich SJ. Inflammatory cells during wound repair: the good, the bad and the ugly. Trends Cell Biol 2005;15:599607.

6. Sheridan RL, Hinson MI, Liang MH, Nackel AF, Schoenfeld DA, Ryan CM, et al. Long-term outcome of children surviving massive burns. JAMA 2000;283:69-73. 